\title{
Population II Stellar Structure and Evolution: A Few Selected Topics
}

\author{
Cesare Chiosi \\ Astronomy Department, University of Padua, Vicolo Osservatorio 5, \\ 35122 Padua, Italy
}

\begin{abstract}
In this review we discuss in some detail the evolution of low mass stars, and other related topics, limiting ourselves to the class of stars with solar and super-solar metal content that likely constitute the basic component of elliptical galaxies and bulges. In particular, we deal with the stars responsible of the UV flux observed in elliptical galaxies and the role played by the chemical composition and mass loss by stellar wind in producing hot progenies with effective temperatures in the range indicated by the UV data. In this context, we briefly report on recent chemo - spectro - photometric models for elliptical galaxies. In addition to this, we discuss the subject of galaxy ages by means of the ultraviolet properties and narrow band indices. Finally, we briefly report on a new formulation of diffusive mixing in stellar interiors and its effects on the structure and evolution of intermediate- and high-mass stars.
\end{abstract}

\section{Introduction}

In this review, no attempt is made to cover all the topics that could be addressed by a report on the progress made in understanding the structure and evolution of population II stars. Recent reviews on the subject are by Iben \& Renzini (1983, 1984), Hesser (1988), Renzini \& Fusi-Pecci (1988), Iben (1991), VandenBerg (1991), Fusi-Pecci \& Cacciari (1991), and Chiosi et al. (1992) to whom the reader should refer for all details. Rather we have selected a few topics on which most effort has been concentrated over the past few years. These are all related to the evolution of old, low-mass stars of high metal content. Stars of this type are likely to be the major constituents of bulges in nearby galaxies and elliptical galaxies in general, in addition to well known cases of metal-rich clusters in the solar vicinity and Galactic Bulge. The peculiarity of the high metallicity objects is that they do not follow the classical scheme in which the core He-burning phase (HB stars) gets redder and redder at increasing metallicity. Indeed, if a threshold metallicity is passed the core He-burning phase splits in two parts, one near the RGB and another one at high effective temperature (cf. Horch et al. 1992, Fagotto et al. 1994a,b,c). These stars are the right candidates to be the dominant source of UV radiation observed in elliptical galaxies (Burstein et al. 1988). 


\section{Observational hints on metallicities and ages}

Direct determinations of metallicities in elliptical galaxies are still rather limited. Most of the information comes from metal line-strength indices and their radial variations (Carollo et al. 1993, Carollo \& Danziger 1994, Davies et al. 1993) or colour gradients (Schombert et al. 1993). The evidence arises that the metallicities for elliptical galaxies are solar or larger. Furthermore, for bright ellipticals there are also indications that the $\alpha$-elements $(\mathrm{O}, \mathrm{Mg}, \mathrm{Si}$, etc.) are enhanced with respect to $\mathrm{Fe}$. The average $[\mathrm{Mg} / \mathrm{Fe}]$, in particular, exceeds that of the most metal rich stars in the solar vicinity by about $0.2-0.3 \mathrm{dex}$, and the ratio $[\mathrm{Mg} / \mathrm{Fe}]$ is expected to increase with the galactic mass up to this value. Due to their proximity, the metallicity of stars in the Galactic Bulge is less of a problem, because this is currently measured by spectroscopic methods (cf. Rich 1990, McWilliam \& Rich 1994). The mean metallicity is slightly lower than solar and long tails towards the metal-poor $([\mathrm{Fe} / \mathrm{H}]=-1)$ and metal-rich end $([\mathrm{Fe} / \mathrm{H}]=1)$ are also present. High metallicities seem also to be indicated by the color-magnitude diagrams (CMD) of stellar populations in the bulges of nearby Galaxies (M31 and M32). In the case of M32 (Freedman 1989, 1992), these stars are most likely in the RGB and AGB phases and metallicities as high as $[\mathrm{M} / \mathrm{H}]=0.1$ are possible (cf. Bica et al. 1990, 1991). Concerning the age, from the magnitude of the brightest AGB stars in M32, Freedman (1989, 1992) concludes that a fraction of these could be as young as 5 Gyr. This is also supported by the new calibration for the maximum AGB star luminosity as a function of the age and metallicity by Bressan et al. (1994, BCF). Similar conclusions would be obtained looking at the CMDs for M32 by Elston \& Silva (1992) and Davidge \& Jones (1992). Finally, deep photometry and CMDs of selected areas of the Galactic Bulge have been presented by Terndrup (1988), Frogel et al. (1990), Udalski et al. (1993a,b), Holtzman et al. (1993), $\mathrm{Ng}(1994)$, and $\mathrm{Ng}$ et al. (1995), and of clusters and companion fields by Ortolani et al. $(1990,1992$, 1993). Since all these regions are observed across the Galactic Disk, a significant contamination by disk stars is present. Therefore, deciphering the information contained in those CMDs requires models for the star density distribution and laws of extinction along the line of sight. Despite the difficulty, Bertelli et al. (1995) studying the region named Terzan $1(l=357.5 ; b=1.0)$, argue that stars with a large range of metallicity $(0.008<Z<0.08)$ and ages from 15 to $12.5 \mathrm{Gyr}$ are present, and more important that stars of the type named H-HB (see below) are perhaps visible.

\section{UV excess in elliptical galaxies: the data}

Much of the available information is from the study of Burstein et al. (1988). The main points are the following:

(1) All studied ellipticals have detectable flux short-ward of about $2000 \AA$.

(2) There are large galaxy to galaxy differences in the level of the UV flux. The intensity of the UV emission is measured by the colour (1550-V).

(3) The colour (1550-V) correlates with the index Mg2, the velocity dispersion $\log \Sigma$ and the luminosity (mass) of the galaxy. The few galaxies (e.g., NGC 205) in which active star formation is seen do not obey these relations. 
(4) An important constraint is posed by the HUT observations by Ferguson et al. (1991) and Ferguson \& Davidsen (1993) of the UV excess in the bulge of M31. In this case the UV emission shows a drop-off short-ward of about $1000 \AA$ whose interpretation requires that the temperature of the emitting source must be about $25,000 \mathrm{~K}$. Only a small percentage of the $912 \leq \lambda \leq 1200 \AA$ flux can be coming from stars hotter than $30,000 \mathrm{~K}$ and cooler than $20,000 \mathrm{~K}$.

\section{UV Excess in elliptical galaxies: the sources}

Excluding ongoing star formation, the UV excess owes its origin to an old component that gets hot enough to power the integrated spectral energy distribution (ISED) of a galaxy in the far UV regions. Three possible candidates are envisaged (cf. Greggio \& Renzini 1990, BCF):

(1) The classical post asymptotic giant branch (P-AGB) stars (see Bruzual 1992, Bruzual \& Charlot 1993, Charlot \& Bruzual 1991). However, even if a small drop-off can be predicted just long-ward of $912 \AA$ they cannot be the sole source of UV flux because of their high mean temperature (about 100,000 K). Nevertheless, these stars have been detected with HST observations in the nucleus of M31 (Bertola et al. 1995) and can contribute as much as 50\% to the UV light. (2) Very blue horizontal branch (HB) stars of extremely low metallicity (Lee 1994). These stars have effective temperatures hotter than about $15,000 \mathrm{~K}$ but much cooler than those of the P-AGB stars. Therefore, depending on their actual effective temperature, they can generate ISEDs in agreement with the observational data. However, most likely they are not the dominant source of the UV flux because the analysis by BCF clarifies that in the wavelength interval $2000<\lambda<3000 \AA$ the ISEDs of the bulge of M31 and of elliptical galaxies like NGC 4649 are fully consistent with the notion that virtually no stars with metallicity lower than $Z=0.008$ ought to exist in the mix of these stellar populations.

(3) Finally, the hot horizontal branch (H-HB) and AGB-manque stars of very high metallicity (say $Z>0.07$ ) which are expected to be present albeit in small percentages in the stellar content of bulges and elliptical galaxies in general. Indeed, these stars have effective temperatures in the right interval and generate ISEDs whose intensity drops short-ward of about $1000 \AA$ by the amount indicated by the observational data. (Ferguson et al. 1991, Ferguson \& Davidsen 1993).

On the side of the stellar evolution theory, the appearance of the various types of UV sources is governed by several important physical factors, each of which is affected by a certain degree of uncertainty still far from being fully assessed. These are the efficiency of mass loss during the RGB and AGB phases, the enrichment law $\Delta Y / \Delta Z$, and finally for the specific case of P-AGB stars the detailed relation between the initial and final mass of the stars at the end of the AGB phase.

P-AGB stars are always present in the stellar mix of a galaxy. The major problem with these stars is the high effective temperature on which we have already reported, and the precise relation between their mass and the turn-off mass (and hence age). The initial-final mass relationship is not firmly established. The most popular empirical determination is by Weidemann (1987). Matching 
this relation depends on the efficiency of mass loss during the AGB phase and other details of model structure (for instance the initial size of the carbon-oxygen core). In general, relations steeper than Weidemann (1987) would predict more massive P-AGB stars and because of their lifetime smaller UV-fluxes at given turn-off mass. The UV flux increases as the stellar models approach the Weidemann (1987) relation. The response of the UV flux to details of initial-final mass relation (for instance its dependence on the metallicity) is so strong that firm conclusions cannot yet be reached. Suffice to recall that for ages older than about $10 \mathrm{Gyr}$, the whole problem is driven by the initial-final mass relation in the mass range 0.8 to $1.0 M_{\odot}$.

The formation of H-HB and AGB-manque with metallicity $Z \gg Z_{\odot}$ is possible either with low values of the enrichment ratio $(\Delta Y / \Delta Z \simeq 1)$ and strong dependences of the mass-loss rates on the metallicity (Greggio \& Renzini 1990) or even with canonical mass-loss rates and suitable enrichment laws $(\Delta Y / \Delta Z \simeq$ $2.5)$ as in Horch et al. (1992) and Fagotto et al. (1994a,b,c). These topics are briefly examined below.

\subsection{Does the mass loss rate from RGB stars increase with the metallicity?}

Studying the stars in the red clump of the old open cluster M67, Tripicco et al. (1993) argue that the rate of mass loss along the RGB increases with the metallicity above the value holding for Globular Clusters and predicted by the classical Reimers (1975) relation. Assuming the current estimates of metallicity (nearly solar), age (about $5 \mathrm{Gyr}$ ), and distance modulus $\left((m-M)_{0}=9.55\right)$ for M67, Tripicco et al. (1993) conclude that the mass of the giant stars in the red clump is about $0.7 M_{\odot}$. Since at the above age, the masses at the turn-off and tip of the RGB are about 1.2 $M_{\odot}$ and $1.3 M_{\odot}$, respectively, it follows that in clusters with nearly solar metallicity, much more mass ought to be lost by the red giant stars than classically predicted. However, Carraro et al. (1995) pointed out that in order to derive the mass of the red stars in the clump from their position in the CMD, the color difference $\Delta(B-V)$ between the turn-off and the base of the RGB should be known with a precision smaller than $\delta[\Delta(B-V)]=0.01$ mag and, at the same time, the distance modulus should be determined with a precision higher than $0.025 \mathrm{mag}$. The analysis of the problem clarifies that in the global isochrone fitting technique, both the distance modulus and mass of the clump stars depend on the above colour difference according to the relations $\partial \mathrm{M}_{\mathrm{V}}^{\mathrm{TO}} / \partial \Delta(B-V) \simeq-9.2$ and $\partial \mathrm{M}^{\mathrm{HB}} / \partial \Delta(B-V) \simeq-22 \mathrm{M}_{\odot} /$ mag. Therefore, an uncertainty in $\Delta(B-V)$ as small as $\delta[\Delta(B-V)]=0.01$ mag implies an uncertainty in the distance modulus of $\delta(m-M)_{o}=0.1 \mathrm{mag}$ and, even more important, an uncertainty in the mass of the clump stars of $0.22 M_{\odot}$. Fits of the CMD of M67 are possible, in which the classical value of the mass for the stars in question is recovered. Therefore, the claim that the rate of mass loss from RGB stars increases with the metallicity is not supported by the present day data for M67. Similar remarks apply to the companion paper by Liebert et al. (1994) on NGC 6791. 


\subsection{Metal-poor, low-mass stars of very old age}

Low-mass, metal-poor stars suffering normal mass loss during the RGB phase, at ages older than about $20 \mathrm{Gyr}$ can undergo the core He-burning phase in the required range of effective temperatures (Lee 1994). The main difficulty with this possibility is the age itself which is much older than commonly assigned to globular clusters (13 \pm 2 Gyr, Fusi-Pecci \& Cacciari 1991). In addition to this, if the UV source resides in the HB stars of low metallicity, the question arises whether their luminosity and relative frequency are compatible with the observed ISED of elliptical galaxies in the range $2000<\lambda 3500 \AA$. Indeed, the analysis of BCF pointed out that the observed ISEDs hint that only very few stars with metallicity lower than about $\mathrm{Z}=0.008$ ought to exist (the analogue of the G-Dwarf Problem in the solar vicinity). In contrast, as the luminosity of the HB stars of low metallicity is significantly fainter than the corresponding one of H-HB and AGB manque stars (see Fagotto et al. 1994a,b,c), the relative number of such stars required to produce the total amount of UV flux could exceed the limit set by the ISED.

\subsection{Enrichment Law $\Delta Y / \Delta Z$}

The law of enrichment $\Delta Y / \Delta Z$ is highly uncertain (Pagel 1989, Pagel et al. 1992). Equally, for its temporal behaviour. With $\Delta Y / \Delta Z=2.5$ (the solar case) and normal rates of mass loss for the RGB phase $(\eta \simeq 0.35-0.45)$, the appearance of the first generation of H-HB and AGB manqué stars in single stellar population occurs at the age of about 7.6 Gyr. This occurs at even younger ages if $\Delta Y / \Delta Z$ is higher than 2.5 (see Fagotto 1994 for details).

\section{New chemo-spectro-photometric models of elliptical galaxies}

BCF and Tantalo et al. (1995, TCBF) constructed new chemo - spectro - photometric models of elliptical galaxies particularly designed to match the colourmagnitude relation (CMR), cf. Bower et al. (1992a,b), and to provide a robust explanation for the UV flux and its dependence on the galactic luminosity (and hence mass), the index $M g_{2}$, and $\log \Sigma$. No details of these models are given here for the sake of brevity. They can be found in BCF and TCBF. Suffice to recall that BCF made use of the closed-box approximation, whereas TCBF adopted the infall description. In both cases the enrichment law is $\Delta Y / \Delta Z=2.5$ and the mass-loss rates are normal ( $\eta=0.35$ in BCF and $\eta=0.45$ in TCBF). Finally, the models allow for galactic winds halting star formation. Galactic winds are at the base of the current interpretation of the CMR for elliptical galaxies (Bower et al. 1992a,b).

The models by BCF and TCBF show that the H-HB and AGB-manque stars are the most plausible candidates as dominant source of UV radiation. Their occurrence is possible in galaxies of sufficiently high mass, which in virtue of the very efficient star formation rate possess higher values both for the mean and peak metallicity. According to the BCF and TCBF models, a few percent of the mass in stars stored in the highest metallicity bins can generate the desired amount of UV flux. In contrast galaxies of lower mass and lower mean and peak metallicity in turn, are not able to generate this type of stars and their UV flux is 
simply produced by their PN component. A natural result of this scheme is that the correlation of the UV flux with the $M g_{2}$ index and the velocity dispersion can be easily accounted for (see BCF and TCBF).

\section{The UV Excess as an Age Indicator}

BCF have suggested that the uprise in the UV flux at the onset of the H-HB and AGB manqué stars can be used as probe of galaxy ages. Since these stars are expected to appear at the rest-frame age of about 7.6 Gyr for $\Delta Y / \Delta Z=2.5$, a sudden change in the $(1550-\mathrm{V})$ colour is expected to be observable at relatively low red-shifts, perhaps reachable with the present day space instrumentation, thus allowing us to date a galaxy from the stellar clock running in the constituent stellar populations. The precise value of the red-shift corresponding to the above age depends on the particular model of the Universe in usage, namely the choice made for the Hubble constant $H_{0}$, the deceleration parameter $q_{0}$, and finally the red-shift of galaxy formation $z_{f o r}$. See BCF and TCBF for all details.

\section{Is there a real age range among elliptical galaxies ?}

The merge of the recent work on population synthesis in narrow band photometry $\left(H_{\beta}, M g_{2},<M g F e>\right.$ indices) by Gonzales (1993), Worthey (1992), and Worthey et al. (1994) and the models for elliptical galaxies in broad band photometry by BCF and TCBF allows us to tackle the question whether or not elliptical galaxies span a large range of ages. This problem has been addressed by Gonzales (1993) by means of the evolutionary path of single stellar populations (SSP) in the so-called $H_{\beta}$ versus $\langle M g F e>$ plane. Galaxies are seen to fall in a rather narrow band comprised between evolutionary paths of SSPs with nearly solar metallicity (narrow range of $\langle M g F e\rangle$ ) and intersect lines of different age. For the sake of illustration, M32 is at the young age side, while NGC4649 is at the old age side. Since the analysis was made using SSPs of given composition instead of galaxy models with ample range of metallicities, the above finding was subjected to the obvious criticism that in real galaxies it would not be possible to disentangle age from metallicity effects.

Bressan et al. (1995, BCT) included the narrow band photometry in the models for elliptical galaxies of BCF and TCBF and found that the galaxy paths run almost parallel to those of the SSPs and that all galaxies of the Gonzales (1993) sample are matched by models whose stellar contents have mean metallicity slightly above solar and may contain peak values up to $\mathrm{Z}=0.08$. Analyzing the properties of the sample galaxies in the space of the parameters $H_{\beta},<M g F e>,(1550-\mathrm{V}), \log \Sigma$, and broad band colours such as (U-V), (B-V), (V-K), etc., BCT reached the following conclusions:

(1) The observed distribution of galaxies in the $H_{\beta}$ versus $\langle M g F e>$ plane is not a sequence of metallicity with bluer galaxies significantly more metal-poor than the red ones. Metallicity effects are expected to broaden the distribution mainly along the $<M g F e>$ axis.

(2) The observed distribution of galaxies in the $H_{\beta}$ versus $\langle M g F e\rangle$ plane is not a mere sequence of age with bluer galaxies significantly younger than the red ones. Although some scatter in the age is possible, it is not likely to be as 
large as indicated by the formal match of data with isochrones in this diagram. M32 in particular according to its $H_{\beta}$ should have an age of about $3 \mathrm{Gyr}$, in disagreement with its $(1550-\mathrm{V})=4.5$ for which much older ages are needed even considering all the uncertainties in the final-initial mass relationship.

(3) The observed distribution of galaxies in the $H_{\beta}$ versus $<M g F e>$ plane does not agree with the expectation from the CMR of Bower et al. (1992a,b) on the notion that it represents a mass-metallicity sequence of nearly coeval objects, in which star formation took place in an initial episode. Most likely, the history of star formation was more complicated than this simple scheme.

(4) The observed $(1550-\mathrm{V})$ colours are not compatible with ages younger than about $7 \mathrm{Gyr}$. UV excesses stronger than $(1550-\mathrm{V})=5.5$ at younger ages are possible only if the age is younger that $1-2 \mathrm{Gyr}$. However, in such a case the remaining UBV colours would be too blue compared with the observational data. Most likely, all galaxies in the sample are older than at least 7 Gyr.

(5) For the subgroup of galaxies whose $(1550-\mathrm{V})$ colours are compatible with old ages (say about 13-15 Gyr), there seems to be a certain spread in age which roughly amounts to about 3 Gyr consistently with the small scatter of the CMR. (6) With the kind of modelling in use, i.e., initial episode of star formation interrupted by galactic, the difficulties encountered with the interpretation of the $\mathrm{H}_{\beta},\langle\mathrm{MgFe}\rangle,(1550-\mathrm{V})$, and $\log \Sigma$ data can be removed by invoking later episodes of star formation in a sort of burst mode. However, the major problem with this suggestion and the notion of coevality, is that some mechanism synchronizing the bursts of star formation should be required in order to dislocate galaxies from the locus in the $H_{\beta}$ versus $\langle M g F e>$ plane consistent with the CMR of coeval objects (CMR baseline) to their actual position. Indeed, even if the statistics is limited, no galaxies are seen along the CMR baseline but for those at the red end.

(7) There is a interesting scenario emerging from this analysis. In brief, galaxies of high velocity dispersion (and hence mass) tend to be confined to the red end of the $H_{\beta}$ versus $\langle M g F e\rangle$ distribution. Their properties are consistent with being old objects (some scatter in the age is perhaps possible) that are able to exhaust the star forming process at very early epochs with no need for later episodes of stellar activity. In contrast, galaxies of lower velocity dispersion (and hence mass) have more contrasting properties. In these systems it seems as if star formation, following the initial activity most likely at epochs as old as in other galaxies, later went through a series of episodes taking place at different epochs that vary from galaxy to galaxy. Equivalently, it could be that star formation (in the nucleus) continued perhaps at minimal levels over significantly longer periods of time. This would mimic a sort of age sequence.

(8) The idea that the overall duration of the star forming activity is inversely proportional to the galaxy mass does not contradict the current information of abundance ratios in elliptical galaxies (Carollo et al. 1993, Carollo \& Danziger 1994, Matteucci 1994). Indeed it would be consistent with the expected trend for the ratio $[\mathrm{Mg} / \mathrm{Fe}]$ inferred from the narrow band indices according to which the average $[\mathrm{Mg} / \mathrm{Fe}]$ in giant elliptical galaxies exceeds that of the most metal rich stars in the solar vicinity by about 0.2-0.3 dex. Furthermore, the ratio $[\mathrm{Mg} / \mathrm{Fe}]$ is expected to increase with the galactic mass up to this value. Matteucci (1994) argues that a possible explanation of this trend could an efficiency 
of star formation increasing with the galactic mass. As a consequence of this, massive ellipticals should have formed on shorter time scales than smaller ellipticals as suggested by the analysis of the parameters $H_{\beta},\langle M g F e\rangle,(1550-\mathrm{V})$, and $\log \Sigma$.

\section{A new model of diffusive mixing in stellar models}

In this section we briefly report on a new formulation of diffusive mixing in stellar interiors proposed by Deng (1994) and Deng et al. (1995a,b). It is aimed to cast light on the kind of mixing that should take place in the so-called overshoot regions surrounding fully convective zones (inner cores, external envelopes and intermediate shells). In these layers, instead of assuming instantaneous and fully efficient mixing, a diffusive scheme is adopted. The algorithm makes use of the so-called scale length most effective for mixing expressed as $l_{d}=P_{d i f} \times 10^{-5} l_{0}$, where $l_{0}$ is the largest scale in the unstable region in units of the local pressure scale height $H_{P}$, and finally $P_{d i f}$ is a fine tuning parameter of the order of unity. Furthermore, it takes two important phenomena of turbulence into account. They are intermittence and stirring, whose occurrence is known from laboratory fluid dynamics (see Deng et al. 1995a,b for more details). The major difference with respect to standard stellar models is that, while fully unstable regions turn out to be completely homogenized by the diffusive algorithm in use, this is not the case for the overshoot regions which undergo partial mixing and build up smooth chemical profiles.

The analysis by Deng et al. (1994a) shows that $P_{\text {dif }}=0.4$ leads to stellar models able to match a number of properties of massive and intermediate mass stars. The key feature of these models is that they possess at the same time evolutionary characteristics that are separately typical of models calculated with different schemes of mixing. In other words, they share the same properties of models with standard overshoot, namely a wider main sequence band, higher luminosity, and longer lifetimes, but also perform extended loops that are the main signature of the semiconvective description of convection at the border of the core.

With the aid of these stellar models, Deng et al. (1995b) analyze the CMDs of supergiant stars in the LMC (Fitzpatrick \& Garmany 1990) and the Milky Way (Blaha \& Humphreys 1989). Particular attention is paid to the star counts across the CMD, the many stars in the Hertzsprung-Russell gap and the related suspected widening of the main sequence band, the so-called Ledge, the ratio of blue to red supergiants $N_{B} / N_{R}$, the location of Wolf-Rayet stars (WR), and the blue progenitor of the SN1987A. It is found that taking into account a plausible scatter in the chemical composition of the supergiant stars in the samples, the many stars in the gap and the Ledge can be easily accounted for. However, problems remain as far as the number frequencies of stars among the various spectral types, the possibility that the main sequence band can extend to spectral types up to B3, the ratio $N_{B} / N_{R}$, and finally the location of WR stars in the CMD. It is suggested that a distinct evolutionary scenario must be invoked for this type of stars (Deng et al. 1995c). Finally, the blue progenitor of SN1987A is not matched by the models, even if clues are found within the same evolutionary scheme for a possible interpretation. 
Acknowledgments. This study has been financed by the Italian Ministry of University, Scientific Research and Technology (MURST) and the Italian Space Agency (ASI).

\section{References}

Bertelli, G., Bressan, A., Chiosi, C., et al. 1995, A\&A, in press Bertola, F., Bressan, A., Burstein, D., et al. 1995, ApJ, 438, 680

Bica, E., Alloin, D., \& Schmidt, A. 1990, A\&A, 228, 23

Bica, E., Barbuy, B., \& Ortolani, S. 1991, ApJ, 382, L15

Blaha, C., \& Humphreys, R. M. 1989, AJ, 98, 1598

Bower, R. G., Lucey, J. R., \& Ellis, R. S. 1992a, MNRAS, 254, 589

Bower, R. G., Lucey, J. R., \& Ellis, R. S. 1992b, MNRAS, 254, 601

Bressan, A., Chiosi, C., Fagotto, F. 1994, ApJS, 94, 63, BCF

Bressan, A., Chiosi, C., Tantalo, R. 1995, A\&A, submitted, BCT

Bruzual, G. 1992, in The Stellar Populations of Galaxies, B. Barbuy \& A. Renzini, Dordrecht: Kluwer Academic Publishers, 311

Bruzual, G., \& Charlot, S. 1993, ApJ, 405, 538

Burstein, D., Bertola, F., Buson, L. M., Faber, S. M., \& Lauer, T.R. 1988, ApJ, 328,440

Carraro, G., Girardi, L., Bressan, A., \& Chiosi, C. 1995, A\&A, submitted

Carollo, C. M., \& Danziger, I. J. 1994, ESO preprint no. 1003

Carollo, C. M., Danziger, I. J., \& Buson, L. 1993, MNRAS, 265, 553

Charlot, S., \& Bruzual, G. 1991, ApJ, 367, 126

Chiosi, C., Bertelli, G., \& Bressan, A. 1992, ARA\&A, 30, 235

Davidge, T. J., \& Jones J. H. 1992, AJ, 104, 1365

Davies, R. L., Sadler, E. M., \& Peletier, R. F. 1993, MNRAS, 262, 650

Deng, L., Bressan, A., \& Chiosi, C. 1995a, A\&A, submitted

Deng, L., Bressan, A., \& Chiosi, C. 1995b, A\&A, submitted

Deng, L., Bressan, A., \& Chiosi, C. 1995c, A\&A, submitted

Elston, R., \& Silva, D. R. 1992, AJ, 104, 1360

Fagotto, F. 1994, in Stellar Populations, M.S.A.It., in press

Fagotto, F., Bressan, A., Bertelli, G., \& Chiosi, C. 1994a, A\&AS, 100, 647

Fagotto, F., Bressan, A., Bertelli, G., \& Chiosi, C. 1994b, A\&AS, 104, 365

Fagotto, F., Bressan, A., Bertelli, G., \& Chiosi, C. 1994c, A\&AS, 105, 39

Ferguson, H. C., \& Davidsen, A. F. 1993, ApJ, 408, 92

Ferguson, H. C., Davidsen A. F., Kriss G. A., et al. 1991, ApJ, 382, L69

Fitzpatrick, E. L., \& Garmany, C. D. 1990, ApJ, 363, 119

Fusi-Pecci, F., \& Cacciari, C. 1991, in New Windows to the Universe, F. Sanchez \& M. Vasquez, Cambridge: Cambridge Univ. Press, 364

Freedman, W. L. 1989, AJ, 98, 1285

Freedman, W. L. 1992, AJ, 104, 1349 
Frogel, J. A., Terndrup, D. M., Blanco, V. M., \& Whitford, A. E. 1990, ApJ, 353,494

Gonzales, J. J. 1993, Ph.D. Thesis, Univ. California, Santa Cruz

Greggio, L., \& Renzini, A. 1990, ApJ, 364, 35

Hesser, J. E. 1988, in Progress and Opportunity in Southern Hemisphere Optical Astronomy, V. M. Blanco \& M. M. Philip, Astron. Soc. Pac. Conf. Ser. vol. 1, Provo: Brigham Young Univ., 161

Holtzman, J. A., Light, R. M., Baum, W. A., et al. 1993, AJ, 106, 1826

Horch, E., Demarque, P., \& Pinsonneault, M. 1992, ApJ, 388, L53

Iben, I. Jr. 1991, ApJS, 76, 55

Iben, I. Jr., \& Renzini, A. 1983, ARA\&A, 21, 271

Iben, I. Jr., \& Renzini, A. 1984, Phys. Rep. 105 (6), 329

Lee, Y-W. 1994, ApJ, 423, 248

Liebert, J., Saffer R. A., \& Green, E. M. 1994, AJ, 107, 1408

Matteucci, F. 1994, A\&A, 288, 57

McWilliam, A., \& Rich, R. M. 1994, ApJS, 91, 749

$\mathrm{Ng}$, Y. K. 1994, Ph.D. Thesis, University of Leiden, the Netherlands

Ng, Y. K., Bertelli, G., Bressan, A., \& Chiosi, C. 1995, A\&A, in press

Ortolani, S., Barbuy, B., \& Bica, E. 1990, A\&A, 236, 362

Ortolani, S., Bica, E., \& Barbuy, B. 1992, A\&AS, 92, 441

Ortolani, S., Bica, E., \& Barbuy, B. 1993, ApJ, 408, L29

Pagel, B. E. J. 1989, in Evolutionary Phenomena in Galaxies, J. Beckmam, \& B. E. J. Pagel, Cambridge: Cambridge University Press, 201

Pagel, B. E. J., Simonson, E. A., Terlevich, R. J., \& Edmunds, M.G. 1992, MNRAS, 255, 325

Reimers, D. 1975, Mém. Soc. Roy. Sci. Liege, $6^{e}$ Ser., 8, 369

Renzini, A., Fusi-Pecci, F. 1988, ARA\&A, 26, 199

Schombert, J. M., Halan, P. C., Barsony, M., \& Rakos, K. D. 1993, AJ, 106, 923

Terndrup, D. M. 1988, AJ, 96, 884

Tantalo, R., Chiosi, C., Bressan, A., \& Fagotto, F. 1995, A\&A, submitted, TCBF

Tripicco, M. J., Dorman B., \& Bel, R. A. 1993, AJ, 106, 618

Udalski, A., Szymański, M., Kałużny, J., et al. 1993a, Acta Astron., 43, 289

Udalski, A., Szymański, M., Kałużny, J., et al. 1993b, Acta Astron., 43, 69

VandenBerg, D. A. 1991, in The Formation and Evolution of Star Clusters, A.S.P. Conf. Ser. vol. 13, Provo: Brigham Young Univ., 183

Weidemann, V. 1987, A\&A, 188, 74

Worthey, G. 1992, Ph.D. Thesis, Univ. California, Santa Cruz

Worthey, G, Faber, S. M., Gonzales, J. J., \& Burstein D. 1994, ApJS, 94, 687 


\section{Discussion}

Roxburgh: I didn't quite understand the model you have of convective diffusion. What one seems to get from numerical simulation is an overshoot region with conditions little different from the unstable region then a very rapid decay of convective velocities. 